\title{
BEAT PERCEPTION IN A NON-VOCAL LEARNER: RATS CAN IDENTIFY ISOCHRONOUS BEATS
}

\author{
Alexandre Celma-Miralles ${ }^{* 1}$, and Juan M. Toro ${ }^{1,2}$ \\ *Corresponding Author: alexandre.celma@upf.edu \\ ${ }^{1}$ Center for Brain and Cognition - ETIC, Universitat Pompeu Fabra, Barcelona, Spain \\ ${ }^{2}$ Institució Catalana de Recerca i Estudis Avançats, Barcelona, Spain
}

The origins of rhythm in music and language seem to be intertwined and may share evolutionary pathways (Fitch, 2006, 2013; Patel, 2006, 2010; Ravignani and Madison, 2017). The development of song in birds and speech in humans follows similar sensorimotor phases and parallel periods of vocal learning (Marler, 1970, 1976), an ability that allows animals to imitate and modify the vocalizations learned from other individuals thanks to specific neural connections (Jarvis, 2006, 2007). For the last decade, the vocal learning beat perception and synchronization hypothesis (Patel, 2006; Patel, Iversen, Bregman, and Schulz, 2009; Schachner, Brady, Pepperberg and Hauser, 2009) has been widely accepted among researchers, proposing that only vocal learners can extract and entrain to a periodic pulse. However, current animal studies reported beat entrainment (Cook, Rouse, Wilson and Reichmuth, 2013) and auditory synchronization (Hattori, Tomonaga and Matsuzawa, 2013) in not classically classified vocal learners, and non-human primates display some rhythmic behaviors in social interactions (see Merchant and Honing, 2013; Ravignani, Gingras, Asano, Sonnweber, Matellán and Fitch, 2013).

To explore to what extent beat perception appears in non-vocal learners, we tested rats (Rattus norvegicus) in a go-no go paradigm. Several studies on comparative research have successfully used this paradigm with rats to discern what components of music and language are actually shared with other species (Crespo-Bojorque and Toro, 2015; de la Mora, Nespor and Toro, 2013). In the current study, thirty-two rats were trained to distinguish between isochronous and non-isochronous beats at four different tempi (Inter Onset Interval of 300, $400,500$ and $600 \mathrm{~ms})$. Forty sequences of twelve pure tones were presented to the rats in each session, and those sequences that were isochronous were reinforced with pellets. For each reinforced isochronous stimulus there was a unique non-isochronous stimulus lasting the same time and comprising the same number of beats in an irregular pseudorandom pattern (see Figure 1). The 
deviant stimuli were never reinforced. Each standard-deviant couple had always the same temporal lapse between the first beat of the sequence and the last one.

(a) Standard isochronous

(b) Deviant non-isochronous

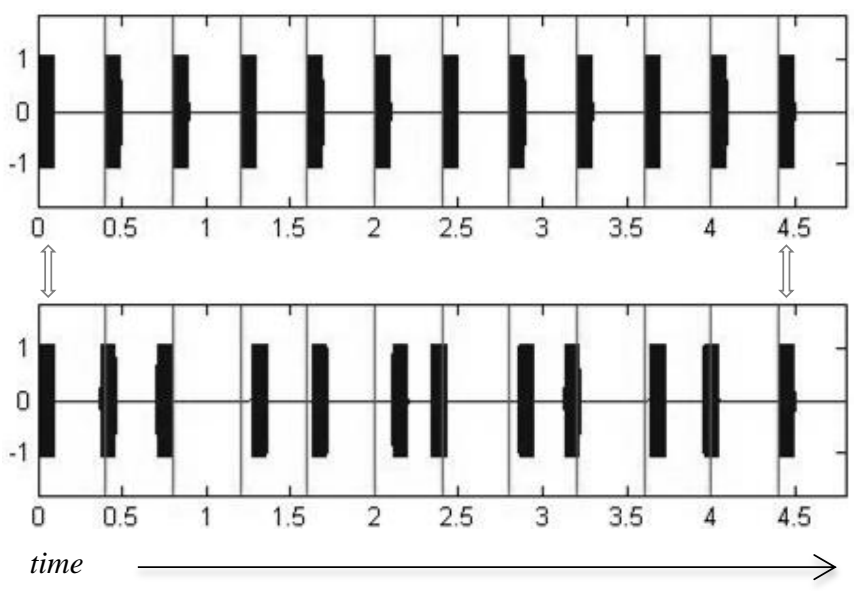

Figure 1. An example of (a) an isochronous stimulus and (b) its non-isochronous counterpart.

After the training sessions, rats were tested three times with ten isochronous stimuli at two new tempi (IOI of 350 and $550 \mathrm{~ms}$ ) and their non-isochronous counterparts. During the tests sessions, rats never received reward for the new isochronous and non-isochronous stimuli. A 2-way repeated measures ANOVA with the within-factors Test (T1, T2, T3) and Stimulus (Standard, Deviant) revealed two significant main effects: the nose-poking behavior of rats increased at each test and was significantly higher for the standard stimuli. In other words, rats insisted more on receiving the pellets over time and succeeded in distinguishing the isochronous beats from the non-isochronous ones.

This first approach to beat perception suggests that the perceptual timing mechanisms underlying isochrony detection are not limited to vocal learners. This is in line with studies on regularity detection in birds (Spierings and ten Cate, 2016; ten Cate, Spierings, Hubert and Honing, 2016) and the neural responses to the beat found in anaesthetized gerbils (Rajendran, Harper, GarciaLazaro, Lesica and Schnupp, 2017). Next studies will focus on more complex metrical rhythms, similar to those used with the mimicking starlings (Hulse, Humpal and Cynx, 1984), to see whether rats are able to deal with beat perception and meter induction beyond the level of isochrony. 


\section{Acknowledgements}

This work was supported by the European Research Council (ERC) Starting Grant agreement n.312519 and by the Spanish Ministerio de Economía y Competitividad (MEC) FPI grant BES-2014- 070547.

\section{References}

Cook, P., Rouse, A., Wilson, M., \& Reichmuth, C. (2013). A California sea lion (Zalophus californianus) can keep the beat: Motor entrainment to rhythmic auditory stimuli in a non vocal mimic. Journal of Comparative Psychology, 127(4), 412.

Crespo-Bojorque, P., \& Toro, J. M. (2015). The use of interval ratios in consonance perception by rats (Rattus norvegicus) and humans (Homo sapiens). Journal of Comparative Psychology, 129(1), 42.

Fitch, W. T. (2006). The biology and evolution of music: A comparative perspective. Cognition, 100(1), 173-215.

Fitch, W. T. (2013). Rhythmic cognition in humans and animals: distinguishing meter and pulse perception. Frontiers in systems neuroscience, 7.

Hulse, S. H., Humpal, J., \& Cynx, J. (1984). Discrimination and generalization of rhythmic and arrhythmic sound patterns by European starlings (Sturnus vulgaris). Music Perception: An Interdisciplinary Journal, 1(4), 442-464.

Hattori, Y., Tomonaga, M., \& Matsuzawa, T. (2013). Spontaneous synchronized tapping to an auditory rhythm in a chimpanzee. Scientific reports, 3, 1566.

Jarvis, E. D. (2006). Selection for and against vocal learning in birds and mammals. Ornithological Science, 5(1), 5-14.

Jarvis, E. D. (2007). Neural systems for vocal learning in birds and humans: a synopsis. Journal of Ornithology, 148(1), 35-44.

Marler, P. (1970). Birdsong and speech development: Could there be parallels? There may be basic rules governing vocal learning to which many species conform, including man. American scientist, 58(6), 669-673.

Marler, P. (1976). An ethological theory of the origin of vocal learning. Annals of the New York Academy of Sciences, 280(1), 386-395.

Merchant, H., \& Honing, H. (2013). Are non-human primates capable of rhythmic entrainment? Evidence for the gradual audiomotor evolution hypothesis. Frontiers in Neuroscience, 7.

De la Mora, D., Nespor, M., \& Toro, J. M. (2013). Do humans and nonhuman animals share the grouping principles of the iambic-trochaic law?. Attention, Perception, \& Psychophysics, 75(1), 92-100.

Patel, A. D. (2006). Musical rhythm, linguistic rhythm, and human evolution. Music Perception: An Interdisciplinary Journal, 24(1), 99-104.

Patel, A. D., Iversen, J. R., Bregman, M. R., \& Schulz, I. (2009). Experimental evidence for synchronization to a musical beat in a nonhuman animal. Current biology, 19(10), 827-830. 
Patel, A. D. (2010). Music, language, and the brain. Oxford university press.

Ravignani, A., \& Madison, G. (2017). The paradox of isochrony in the evolution of human rhythm. Frontiers in psychology, 8.

Ravignani, A., Gingras, B., Asano, R., Sonnweber, R., Matellán, V., \& Fitch, W. T. (2013). The Evolution of Rhythmic Cognition: New Perspectives and Technologies in Comparative Research. In the Proceedings of the 35th Annual Conference of the Cognitive Science Society, Berlin, Germany, 31 July-3 August.

Rajendran, V. G., Harper, N. S., Garcia-Lazaro, J. A., Lesica, N. A., \& Schnupp, J. W. (2017, November). Midbrain adaptation may set the stage for the perception of musical beat. Proceedings of the Royal Society B, Vol. 284, No. 1866, p. 20171455. The Royal Society.

Schachner, A., Brady, T. F., Pepperberg, I. M., \& Hauser, M. D. (2009). Spontaneous motor entrainment to music in multiple vocal mimicking species. Current Biology, 19(10), 831-836.

Spierings, M. J., \& ten Cate, C. (2016). Zebra finches as a model species to understand the roots of rhythm. Frontiers in neuroscience, 10.

ten Cate, C., Spierings, M., Hubert, J., \& Honing, H. (2016). Can birds perceive rhythmic patterns? A review and experiments on a songbird and a parrot species. Frontiers in psychology, 7. 